\title{
Modeling Transverse Dispersion and Variable Density Flow in Porous Media
}

\author{
H. M. Nick • R. Schotting • M. Gutierrez-Neri • \\ K. Johannsen
}

Received: 27 May 2008 / Accepted: 14 August 2008 / Published online: 11 September 2008

(C) The Author(s) 2008. This article is published with open access at Springerlink.com

\begin{abstract}
A two-dimensional numerical model is used to study the nonlinear behavior of density gradients on transverse dispersion. Numerical simulations are conducted using $d^{3} f$, a computer code for simulation of density-dependent flow in porous media. Considering a density-stratified horizontal flow in a heterogeneous porous media, a series of simulations is carried out to examine the effect of the density gradient on macro-scale transverse dispersivity. Changing salt concentration significantly affects fluid properties. This physical behavior of the fluid involves a non-linearity in modeling the interaction between salt and fresh water. It is concluded that the large-scale transport properties for high density flow deviate significantly from the tracer case due to the spatial variation of permeability, described by statistical parameters, at the local-scale. Indeed, the presence of vertical flow velocities induced by permeability variations is responsible for the reduction of the mixing zone width in the steady state in the case of a high density gradient. Uncertainties in the model simulations are studied in terms of discretization errors, boundary conditions, and convergence of ensemble averaging. With respect to the results, the gravity number appears to be the controlling parameter for dispersive flux. In addition, the applicability and limitations of the nonlinear model of Hassanizadeh (1990) and Hassanizadeh and Leijnse (1995) (Adv Water Resour 18(4):203-215, 1995) in heterogeneous porous media are investigated. We found that the main cause of the nonlinear behavior of dispersion, which is the interaction between density contrast and vertical velocity, needs to be explicitly accounted for in a macro-scale model.
\end{abstract}

Keywords Heterogeneity · Porous media - Density contrast - Solute transport . Macro-dispersivity

H. M. Nick $(\bowtie)$

Department of Earth Sciences and Engineering, Imperial College, London, UK

e-mail: h.maghami-nick07@imperial.ac.uk

H. M. Nick · R. Schotting · M. Gutierrez-Neri

Environmental Hydrogeology Group, Department of Earth Sciences, University of Utrecht, Utrecht, The Netherlands

K. Johannsen

The Bergen Center for Computational Science, Bergen, Norway 


\section{Introduction}

Fluid density variation occurs in some specific hydrological situations due to the change in fluid properties, including solute concentration, temperature, and pressure. Varieties of studies have addressed the effect of density contrasts on flow and transport in porous media; see, e.g., Diersch and Kolditz (2002). Flow of groundwater close to salt domes, sea water intrusion, geothermal flow, liquid waste disposal flow, and seepage from waste disposal sites are typical examples. Groundwater in the vicinity of salt domes contains large amounts of dissolved salt, up to the saturation limit. There exists convincing experimental evidence that the dispersive behavior of salt water in a porous medium is affected by the presence of high-salt-concentration gradients.

Natural porous formations are in general heterogeneous, and display spatial variability of their geometric and hydraulic properties, which can affect the dispersive properties (Bellin et al. 1992; Burr et al. 1994). Many studies have considered how spatial heterogeneity in hydraulic conductivity affects fluid flow and solute transport in porous media (e.g., Chaudhuri and Sekhar 2006). This heterogeneity is usually characterized by a spatial variability of hydraulic conductivity. As a result, the macro-dispersion coefficient describes solute spreading due to local variations in the velocity caused by spatial heterogeneity of conductivity at a smaller scale. This has been studied by Dagan (1989) and Gelhar and Axness (1983).

Longitudinal and transverse dispersivity vary with scale; thus, the spatial variability of dispersivity is generally assumed as scale-dependent dispersion (Gelhar 1986). Many studies have applied numerical and analytical solutions to investigate scale-dependent dispersive transport in a uniform flow field (Pickens and Grisak 1981; Yates 1990). In all cases, the transverse dispersivities are smaller than the longitudinal dispersivities. Moreover, in contrast to longitudinal macro-dispersivity, transverse macro-dispersivity approaches an asymptotic value only slightly larger than the local scale coefficient (Dagan 1988; Cirpka and Attinger 2003). Although the transverse dispersion coefficient is much smaller than the longitudinal dispersion coefficient, it plays a main role in many groundwater transport processes, in particular, when studying the fate of contaminate plumes, see, e.g., Ham et al. (2004).

Several studies on longitudinal hydrodynamic dispersion report experiments where a less dense fluid is displaced by a denser fluid. These studies revealed that an increase in density gradient leads to a reduced mixing zone. Hassanizadeh (1990) and Moser (1995) performed similar stable brine displacement experiments (vertical flow in the column) to study the density contrast effects on longitudinal dispersion. Hassanizadeh (1986) derived extended versions of Darcy's and Fick's laws for situations in which high concentration is present. Hassanizadeh (1990) and Hassanizadeh and Leijnse (1995) suggested a new nonlinear extension of Fick's laws. Their findings are confirmed by Schotting et al. (1999), who analyzed the experiments of Moser (1995) in homogeneous media. They also derived an analytical solution based on the nonlinear theory of Hassanizadeh. This analytical solution is in good agreement with the finding of Diersch and Kolditz (2002). Furthermore, Watson et al. (2002b) confirmed the findings of Hassanizadeh and Leijnse (1995) and Moser (1995).

Recently, numerical experiments conducted by Landman (2005) showed a decrease in the longitudinal dispersivity with increasing gravity number and for a small log-permeability variance. The results are in accordance with the laboratory experiments of Moser (1995) and Jiao and Hotzl (2004), which were performed in nearly homogeneous media. Moreover, Landman showed that the relationship between apparent longitudinal dispersivity and gravity number depends on the heterogeneity of the porous medium such that for media with stronger heterogeneity, the dispersivity decreases more slowly than that in weakly heterogeneous 
porous media. Welty and Gelhar (1991) and Egorov et al. (2005) studied a model based on mathematical upscaling of the local scale equations using stochastic and homogenization techniques, respectively. The model of Egorov using a homogenization technique to obtain a macroscopic model for density and viscosity dependent dispersion is similar to the stochastic theory of Welty and Gelhar (1991). The predictions of Egorov's homogenization model are directly comparable to the results of the numerical experiments for weakly heterogeneous porous media, although it is not able to describe the short time behavior of the dispersion (Landman 2005).

Density gradients also have an effect on lateral dispersion. The effect of density differences on transverse dispersion was studied using a horizontal sand box when two stratified miscible fluids are simultaneously injected (Grane and Gardner 1961). They observed that for a highly permeable porous medium, the density differences cause a decrease of the transverse dispersion. The effect of density gradients on transverse dispersion in the horizontal flow (for a stable horizontal density front) was investigated experimentally by Leroy et al. (1992). Similar to what was found for longitudinal dispersion in the vertical flow, a reduction of the mixing zone width was found with increasing density difference. Moreover, this effect was found to be more pronounced at low velocities. Leroy et al. (1992) furthermore reported that the gravity effect is much larger for stratified media than for homogeneous porous media. In addition, the effect of density and viscosity variations on mixing solute due to advection and molecular diffusion was studied mathematically by Thiele (1999). This research is applicable to flows with small Peclet number since the hydrodynamic dispersion mechanism is disregarded. A series truncation method was used to determine the concentration distribution. He observed stronger effect caused by density variations on mixing properties than the viscosity effect. Johannsen et al. (2002) simulated the saltpool benchmark experiments, in which a horizontal density interface is situated in a flow field that is mainly horizontal. In order to match the experimental results, the value of transverse dispersivity had to be reduced considerably for the high concentration (saltpool 2) case. Welty et al. (2003) used stochastic analysis to derive a complex dependency of transverse dispersivity on concentration and concentration gradient which, recently, has been tested by Starke and Koch (2006). Experiments in a large tank were performed to investigate the effect of density gradients on transverse dispersion (Starke 2005). They revealed the dependency of measured transverse dispersivity on concentration gradient and velocity. In their study, decrease of transverse dispersivity with increasing seepage velocity was obtained. In spite of these related studies, effects of density and viscosity contrast on transverse dispersion have not been adequately considered up to now.

\subsection{Objectives and Outlines}

Changing salt concentration significantly affects the fluid properties; density and viscosity of fluid are increased by increasing mass concentration. These physical dependencies imply nonlinearities in modeling the interaction between salt and fresh water.

This study addresses two issues: The effect of density gradients in heterogeneous media on transverse dispersivity, and the validity and applicability of the nonlinear model of Hassanizadeh and Leijnse (1995) for transverse dispersion.

In this article, a simulation procedure is described that contains description of the numerical model as well as a measurement method for transverse dispersivity. This is followed by a discussion of computation errors in terms of discretization error, boundary condition effect, and convergence of averaging procedure. Then, the results of a series of accurate simulations are provided to study the density-driven flow problem in heterogeneous porous media. 
The flow and transport are simulated with several realizations of random permeability fields. Simulation of different density contrasts between fresh and salt water are presented. And the relationship between scaled transverse macro-dispersivity and gravity number in terms of different density difference, flow rate, local transverse dispersivity, and heterogeneity is investigated. Finally, these are used to validate the nonlinear model of Hassanizadeh and Leijnse (1995) for transverse dispersivity in a two-dimensional domain.

\section{Numerical Simulations}

\subsection{The Mathematical Model}

The governing equations are Darcy's law

$$
\mathbf{q}=-\frac{\kappa}{\mu}(\nabla P-\rho \mathbf{g})
$$

where $\mathbf{q}$ is the specific discharge $\left[\mathrm{LT}^{-1}\right], \kappa$ is the intrinsic permeability tensor $\left[\mathrm{L}^{2}\right], \mu$ is the dynamic viscosity $\left[\mathrm{ML}^{-1} \mathrm{~T}^{-1}\right], \nabla P$ is the pressure gradient $\left[\mathrm{ML}^{-1} \mathrm{~T}^{-2}\right], \rho$ is the fluid density $\left[\mathrm{ML}^{-3}\right]$, and $\mathbf{g}$ is the gravity vector $\left[\mathrm{LT}^{-2}\right]$. Moreover, conservation of mass in a non-deformable porous medium yields

$$
n \frac{\partial \rho}{\partial t}+\nabla \cdot(\rho \mathbf{q})=0
$$

and the mass balance for a non-reactive and non-adsorbing solute in a non-deformable porous medium is given by

$$
n \frac{\partial(\rho \omega)}{\partial t}+\nabla \cdot(\rho \mathbf{q} \omega+\mathbf{J})=0
$$

where $\omega$ denotes the solute mass fraction [-], and $n$ denotes the porosity [-]. The dimensionless variable $\omega$ is defined as the ratio of mass concentration $\left[\mathrm{ML}^{-3}\right], C$, to fluid density, i.e., $\omega=C / \rho$. The vector $\mathbf{J}$ is the dispersive mass flux $\left[\mathrm{ML}^{-2} \mathrm{~T}^{-1}\right]$ given by Fick's Law:

$$
\mathbf{J}=-\rho \mathbf{D} \nabla(\omega)
$$

where the hydrodynamic dispersion tensor, D, accounting to Scheidegger (1961), is given by

$$
\mathbf{D}=\left(n D_{\text {eff }}+\alpha_{\mathrm{T}}|\mathbf{q}|\right) \mathbf{I}+\left(\alpha_{L}-\alpha_{\mathrm{T}}\right) \frac{\mathbf{q q}}{|\mathbf{q}|}
$$

Here, $D_{\text {eff }}$ is the effective molecular diffusion in which, $D_{\text {eff }}=D_{\mathrm{m}} / \tau$, where $D_{\mathrm{m}}$ denotes the molecular diffusion coefficient $\left[\mathrm{L}^{2} \mathrm{~T}^{-1}\right]$, and $\tau$ is tortuosity [-]. The parameters $\alpha_{L}$ and $\alpha_{\mathrm{T}}$ are the longitudinal and transverse dispersivities [L], respectively, $\mathbf{I}$ is the unit tensor, and $|\mathbf{q}|$ is the magnitude of the specific discharge vector. In spite of the fact that many physical phenomena can alter the fluid properties, for the sake of simplicity, we consider only the effect of salt concentration on fluid density:

$$
\rho=\rho_{o} \mathrm{e}^{\gamma_{\rho} \omega} .
$$

where the value of $\gamma_{\rho}$ approximately is equal to $\ln (2)$ for $\mathrm{NaCl}$. The aforementioned sets of equations are used to model flow and transport in porous media in the case where the effect of salt mass fraction on water density is considered. 


\subsection{Assumptions}

The following assumptions are imposed to characterize the mixing properties of density dependent flow for a non-adsorbing and non-reactive solute through a two-dimensional isotropic porous medium:

- At the local scale, i.e., the scale of heterogeneities, the standard porous media transport equations are valid, including linear Fick's Law.

- Assuming constant porosity, the heterogeneity is only in the intrinsic permeability. The permeability is regarded as a random field; therefore, at the local scale, velocity is influenced by spatial permeability variations. Considering that the domain under consideration is large compared to the scale of heterogeneities.

- The actual flow in reality is three-dimensional; however, in the current study, the flow is treated as two-dimensional. Note that as the averaging area is increased by using a threedimensional porous medium, the uncertainty in the averaged concentration decreases (Landman 2005).

- Assuming that viscosity variations are small compared to the density effects, viscosity variations are disregarded.

- Molecular diffusion is considered as a constant in density-dependent flow problem.

Note that viscosity and density variations have opposite effects on dispersion in a stable system, i.e., the lighter fluid is on top of the heavier fluid; density contrasts slow down the rate of advancement of dispersion, whereas viscosity contrast enhances it. Thiele (1999) stated that density differences have a large effect on the velocity distribution rather than viscosity contrast in gravity affected transverse dispersion. In addition, we suppose that the ratio between Rayleigh number and Peclet number controls the characteristics of solute mixing in the presence of gravity forces and viscous forces. Therefore, the dimensionless gravity number, $N_{\mathrm{g}}=\mathrm{Ra} / \mathrm{Pe}=\Delta \rho \kappa g / \mu q$, is used to represent the ratio of different forces. Here, $\Delta \rho$ denotes the density contrast.

\subsection{Numerical Codes}

Several numerical codes exist to simulate variable density flow problems, while these do not lend themselves easily to analytical solutions except with simplifications (Simmons 2005). The computer codes $d^{3} f$ and FGEN are used to carry out the simulations since the change of convective motion caused by the heterogeneity in the presence of gravity force is the main concern.

\subsubsection{Density-Dependent Flow Model}

The computer code $d^{3} f$ is employed to simulate density-dependent flow and transport in porous media (Fein 1998, Johannsen 2004). It contains a preprocessor for interactively designing geometry and physical parameters of model problems, a simulator for discretizing and solving the equations, and a postprocessor for visualization and data extraction. The simulator is based on the software package UG, a toolbox for discretizing and solving partial differential equations (Bastian et al. 1997). The postprocessor is based on the software package GRAPE.

The equations of flow and transport in porous media are discretized using a vertex-centered finite volume scheme, where a finite volume is associated with each grid point (node). A consistent velocity approximation is applied (Frolkovic 1998). This code provides a fully mass 
conservative spatial and time discretization. The discretization is locally mass-conserving and second-order consistent for the unknowns: pressure and scaled mass fraction. It uses a finite volume scheme for discretization in space with central differencing. And Euler method (finite differences) is used for time. To solve the discrete equations, a fully coupled implicit solution technique is applied. The nonlinear discrete equations are solved using Newton's method; for the linear sub-problems, a linear multigrid method is applied (Johannsen et al. 2005). Furthermore, a great advantage of this package is its support for parallelism. The calculations are carried out on a MIMD parallel computer using up to 32 processors. The code has been applied to several density-dependent flow problems; see, e.g., Johannsen et al. (2002), Johannsen (2003), Johannsen et al. (2005), and Landman (2005).

\subsubsection{Random Field Generator}

In order to consider heterogeneity in our density-dependent problem, a random field generator, FGEN, developed by Robin et al. (1993), is used to generate the permeability fields.

A number of studies suggest that the probability density function for hydraulic conductivity is log-normal, e.g., Dagan (1989). If the permeability $\kappa$ is log-normally distributed, a new parameter $Y=\ln (\kappa)$ that is normally distributed can be defined. An isotropic Gaussian correlation function for the log-permeability can be used, which is given by:

$$
R(h)=\mathrm{e}^{-\left(\frac{h}{\lambda}\right)^{2}}
$$

where $\lambda$ denotes correlation length, and $h$ denotes Euclidean distance. Therefore, a normal distribution, $Y$, with sample size given by the number of elements in the mesh can be formed with a given mean, $\mu$, and a given variance, $\sigma_{f}^{2}$.

\subsection{Set-Up of the Numerical Experimental}

Transverse dispersivity coefficients are typically evaluated by studying the steady-state transverse concentration profiles of conservative solutes in parallel flow. Usually, two miscible fluids are simultaneously injected into a porous medium, side by side in a horizontal sand box, and the concentration profiles are determined consequently (Grane and Gardner 1961; Starke 2005; Starke and Koch 2006).

The model used in this study is shown in Fig. 1. A stable density stratified system in a two-dimensional porous media is considered where a fluid of lower density overlies a fluid of higher density.

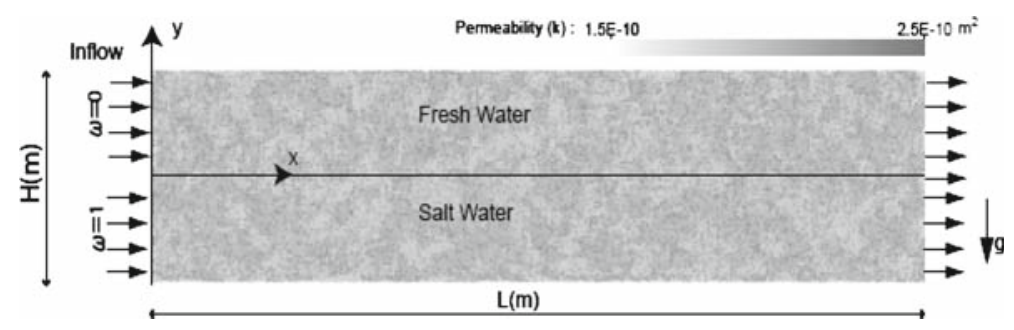

Fig. 1 Numerical experiment set-up 
Table 1 Model parameters

\begin{tabular}{lll}
\hline Height $H(Y$ direction $)$ & 0.4 & $\mathrm{~m}$ \\
Length $L(X$ direction $)$ & 4 & $\mathrm{~m}$ \\
Permeability $\bar{\kappa}$ & $2 \times 10^{-10}, 4 \times 10^{-10} \mathrm{~m}^{2}$ \\
Variance $\sigma_{f}^{2}$ & $0.1,0.25,0.5$ & - \\
Correlation length $\lambda$ & $0.005,0.01,0.015$ & $\mathrm{~m}$ \\
Density contrast $\Delta \rho$ & $0-200$ & $\mathrm{~kg} / \mathrm{m}^{3}$ \\
Velocity $q$ & $2 \times 10^{-5}-1 \times 10^{-4}$ & $\mathrm{~m} / \mathrm{s}$ \\
Viscosity $\mu$ & $1 \times 10^{-3}$ & $\mathrm{~kg} / \mathrm{ms}$ \\
Effective molecular diffusivity $D_{\text {eff }}$ & $1 \times 10^{-9}$ & $\mathrm{~m} / \mathrm{s}$ \\
Longitudinal microscale dispersivity $\alpha_{L} 1 \times 10^{-3}$ & $\mathrm{~m}$ \\
Transverse microscale dispersivity $\alpha_{\mathrm{T}}$ & $1 \times 10^{-4}$ & $\mathrm{~m}$ \\
Porosity $n$ & 0.4 & - \\
\hline
\end{tabular}

\subsubsection{Boundary and Initial Conditions}

We assume a piece-wise constant initial scaled mass fraction distribution according to: $\omega(x, y>0, t=0)=0$ and $\omega(x, y<0, t=0)=1$, where $\omega$ denotes scaled mass fraction. In addition, zero scaled mass fraction flux and pressure as boundary conditions at the top and bottom of the domain are

$$
\begin{aligned}
& (\mathbf{q} \omega-\mathbf{D} \nabla \omega) \cdot \mathbf{n}=0 \\
& \mathbf{q} \omega \cdot \mathbf{n}=0
\end{aligned},
$$

where the vector $\mathbf{n}$ denotes the outer normal vector on boundaries. The left-hand boundary conditions are

$$
\begin{array}{ll}
\omega(0, y)=0 & \forall y \in] 0, H / 2] \\
\omega(0,0)=0.5 & \\
\omega(0, y)=1 & \forall y \in[-H / 2,0[ \\
\mathbf{q} \cdot \mathbf{n}=-q &
\end{array} .
$$

The right-hand boundary conditions are

$$
\begin{aligned}
& \mathbf{D} \nabla \omega \cdot \mathbf{n}=0 \\
& \mathbf{q} \cdot \mathbf{n}=q \\
& P(L, 0)=0
\end{aligned}
$$

The abovementioned setting applies to all our model problems. And the problems change in the choice of velocity, mean permeability, variance, local dispersivity, and density contrast. Table 1 lists the model parameters.

\subsection{Analysis Techniques}

\subsubsection{Apparent Dispersivity}

Equation 3 for the tracer case by virtue of having neither vertical velocity, i.e., $q=q_{x}=$ const., nor longitudinal dispersion, i.e., $\partial^{2} \omega / \partial x^{2}$ is negligible compare to $\partial^{2} \omega / \partial y^{2}$, reduces to

$$
n \frac{\partial \omega}{\partial t}+q \frac{\partial \omega}{\partial x}=D_{\mathrm{T}} \frac{\partial^{2} \omega}{\partial y^{2}}
$$


where $D_{\mathrm{T}}$ denotes transverse dispersion. Assuming $\left\{\begin{array}{ll}\omega=0 & y \rightarrow+\infty \\ \omega=1 & y \rightarrow-\infty\end{array}\right.$, the solution of Eq. 11 for steady state gives

$$
\omega(x, y)=\frac{1}{2} \operatorname{erfc}\left(\frac{y}{2 \sqrt{D_{\mathrm{T}} x / q}}\right)
$$

Hence, this formula can be used to determine the apparent transverse dispersivity, $A_{\mathrm{T}}$, at the macroscale. For homogeneous porous media, fitting this equation gives the same local transverse dispersivity when there are no gravity effects, i.e., the tracer case.

While in heterogeneous porous media (Fig. 2), Eq. 12 cannot fit exactly the scaled mass fraction profiles at each travel distance, $x$, due to the fact that scaled mass fraction profiles at different travel distances are not symmetric, whereas Eq. 12 represents a symmetric curve. The meandering in the shape of the mixing zone is owing to the velocity variations caused by heterogeneities. In order to measure apparent transverse dispersivity, improvement of a measurement method is needed. Moreover, there is a need to gain an average dispersivity coefficient that can explain how more complex and realistic heterogeneity geometries affect variable density flow processes in porous media. According to the dependence of the mixing zone width on both travel distance and transverse dispersion coefficient at each $x$ location, averaging along travel distance $x$ is impossible. To resolve this dilemma, we introduce a new coordinate $(x, \eta)$ to consider the problem as a one-dimensional problem. It is assumed that the ensemble of different realizations produces a scaled mass fraction profile which may be compared to that of homogenous porous media. The new coordinate is defined as follows:

$$
\left\{\begin{array}{l}
x=x \\
\eta=\frac{y}{\sqrt{x}}
\end{array}\right.
$$

Figure 3 illustrates the new coordinate system. Therefore, the Eq. 12 changes to

$$
\omega(x, \eta)=\frac{1}{2} \operatorname{erfc}\left(\frac{\eta}{2 \sqrt{n D_{\text {eff }} / q+A_{\mathrm{T}}}}\right)
$$

It should be noted that the Eq. 14 is the solution of the steady-state tracer transport equation in a semi-infinite domain. The transverse dispersivity calculated, i.e., $A_{\mathrm{T}}$, with this equation, and thus is called "apparent".

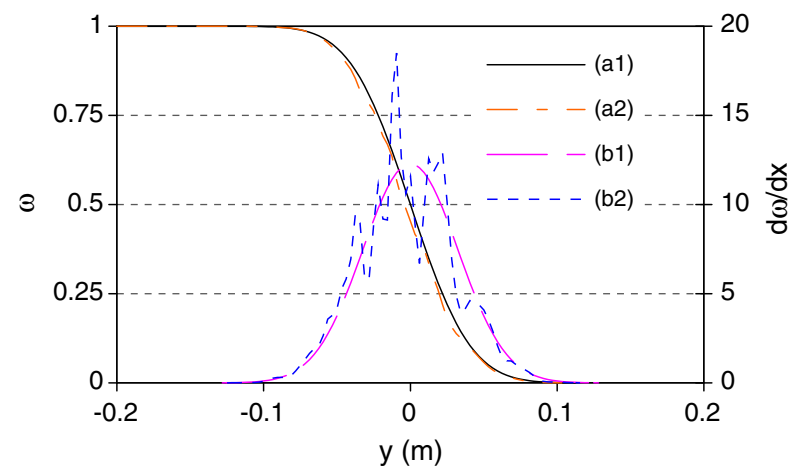

Fig. 2 Scaled mass fraction profiles in a homogeneous (a1) and a heterogeneous (a2) porous medium for tracer case. Due to the heterogeneity of media scaled mass fraction gradient profile (b2) is not symmetric, and unlike scaled mass fraction gradient profile in a homogeneous porous (b1) medium is not Gaussian 


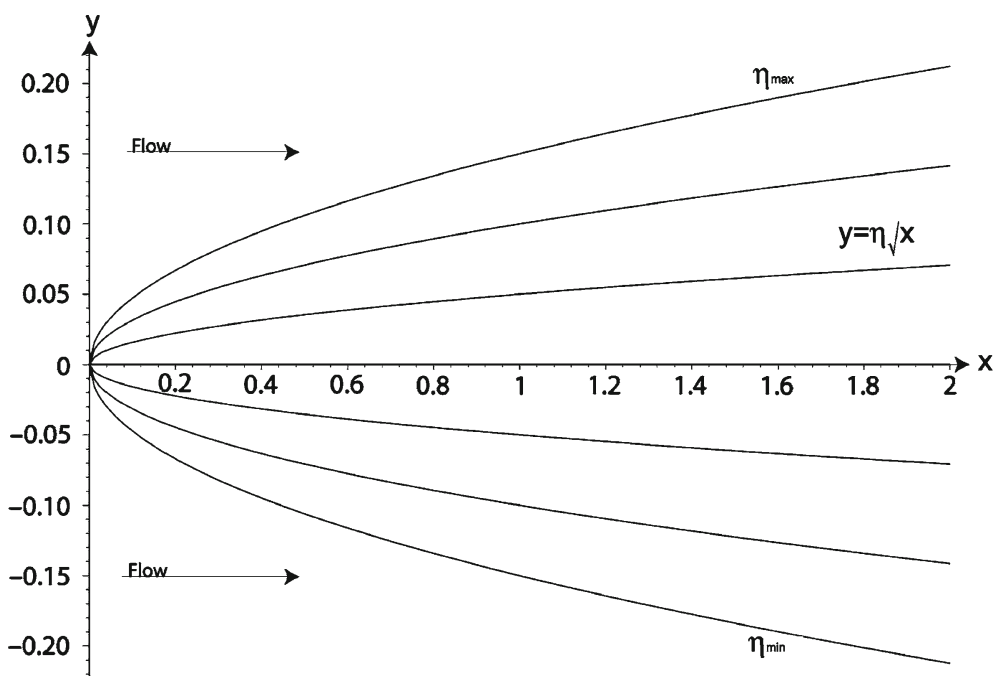

Fig. 3 Iso mass fraction lines in a homogenous porous medium. The new coordinate system $(x, \eta)$ is used for averaging mass fraction values

\subsubsection{Average Scaled Mass Fraction}

The ensemble average of the scaled mass fraction for each mesh node is given by the average over number of realizations, $N$ :

$$
\langle\omega(x, \eta)\rangle=\frac{\sum_{n=1}^{N} \omega(x, \eta)}{N}
$$

The vertical average scaled mass fraction, $\bar{\omega}(x)$, and the variance of a single ensemble profile, $\sigma_{\langle\omega(x)\rangle}^{2}$, at each travel distance, $x$, are determined by

$$
\begin{aligned}
& \bar{\omega}(x)=\frac{1}{\left(\eta_{\max }-\eta_{\min }\right)} \int_{\eta_{\min }}^{\eta_{\max }}\langle\omega(x, \eta)\rangle d \eta \\
& \sigma_{\langle\omega(x)\rangle}^{2}=\frac{1}{N_{\eta}-1} \sum_{n=1}^{N_{\eta}}(\langle\omega(x, \eta)\rangle-\bar{\omega}(x))^{2}
\end{aligned}
$$

where $N_{\eta}$ denotes the number of scaled mass fraction points at each profile, and $\eta_{\max }=$ $-\eta_{\min }=(H / 2) / \sqrt{L}$. The ensemble scaled mass fractions are then averaged over travel distance, $x$, for each $\eta$ value. This averaged mass fraction is defined as

$$
\langle\bar{\omega}(x, \eta)\rangle=\frac{1}{\left(x-x_{1}\right)} \int_{x_{1}}^{x}\langle\omega(x, \eta)\rangle \mathrm{d} x
$$


The vertical average of the ensemble scaled mass fractions, $\langle\varpi(x)\rangle$, and the variance of the average of ensemble profiles, $\sigma_{\langle\varpi(x)\rangle}^{2}$, at each travel distance, $x$, are given by

$$
\begin{aligned}
\langle\varpi(x)\rangle & =\frac{1}{\left(\eta_{\max }-\eta_{\min }\right)} \int_{\eta_{\min }}^{\eta_{\max }}<\bar{\omega}(x, \eta)>d \eta \\
\sigma_{<\varpi(x)>}^{2} & =\frac{1}{N_{\eta}-1} \sum_{n=1}^{N_{\eta}}(<\bar{\omega}(x, \eta)>-<\varpi(x)>)^{2}
\end{aligned}
$$

Average apparent dispersivity, $A_{\mathrm{T}}$, is calculated by fitting Eq. 14 to $<\bar{\omega}(x, \eta)>$ values at each travel distance, $x$. Thus, a curve with a minimal deviation from all data points is desired. This best-fitting curve can be obtained by the method of least squares.

\subsection{Computational Errors}

\subsubsection{Discretization Errors}

In general, discretization errors are the dominant sources of numerical errors in computational simulations (Roy 2005). The numerical solution of both the tracer case and the brine case in a heterogeneous porous medium for steady state are investigated in terms of discretization errors. The parameter values used are $q=2 \times 10^{-5} \mathrm{~m} / \mathrm{s}, \Delta \rho=0,200 \mathrm{~kg} / \mathrm{m}^{3}, \sigma^{2}=0.1$, and $\lambda=0.01$. For convergency study, four different grid levels are considered, see Table 2 .

By conducting calculations on three separate grids and assuming that the results are in the asymptotic regime to use Richardson extrapolation (Oberkampf and Trucano 2002), the empirical order $\tilde{P}_{j}$ of the discretization (order of error reduction or order of accuracy) is

$$
\begin{aligned}
& \tilde{P}_{j}=\frac{\operatorname{Ln}\left(\frac{\left\|\omega^{j-1}-\omega^{j-2}\right\|}{\left\|\omega^{j}-\omega^{j-1}\right\|}\right)}{\operatorname{Ln}(r)} \\
& r=\frac{h^{j}}{h^{j-1}}=\frac{h^{j-1}}{h^{j-2}}
\end{aligned}
$$

The ratio $\left\|\omega^{j-1}-\omega^{j-2}\right\| /\left\|\omega^{j}-\omega^{j-1}\right\|<0$ implies that the three discrete solutions do not converge monotically as the grid is refined. Moreover, $h^{j}$ denotes the grid size corresponding to the grid discretization level $j$. In general, $\omega^{j}$ denotes the solution, e.g., scaled mass fraction, on grid level $j$, and $\|$.$\| denotes the \mathrm{L}^{2}$ norm. The discretization error at grid discretization level $j$ is, in addition, estimated by

$$
D E_{j}=\left\|\omega^{j}-\omega^{\infty}\right\|=\frac{\left\|\omega^{j}-\omega^{j-1}\right\|}{r^{\tilde{P}_{j}}-1} .
$$

Table 2 Four different grids employed in the discretization error analysis

\begin{tabular}{lcl}
\hline Grid level & Grid nodes & Grid spacing, $h(\mathrm{~m})$ \\
\hline 5 & $33 \times 321$ & 0.01248 \\
6 & $65 \times 641$ & 0.00624 \\
7 & $129 \times 1,281$ & 0.00312 \\
8 & $257 \times 2,561$ & 0.00156 \\
\hline
\end{tabular}


Table 3 Discretization error and fraction error of $\omega(x, y)$ on different grid levels $j$ for both the tracer and brine experiments in a heterogeneous porous medium

\begin{tabular}{|c|c|c|c|c|c|c|}
\hline \multirow[t]{2}{*}{ Grid levels } & \multicolumn{3}{|l|}{ Tracer } & \multicolumn{3}{|l|}{ Brine } \\
\hline & $\left\|\omega^{j}-\omega^{j-1}\right\|$ & $D E_{j}$ & $E_{j}(\%)$ & $\left\|\omega^{j}-\omega^{j-1}\right\|$ & $D E_{j}$ & $E_{j}(\%)$ \\
\hline 6 & $1.18 \mathrm{E}-02$ & $2.95 \mathrm{E}-03$ & 1.97 & $8.79 \mathrm{E}-03$ & $3.23 \mathrm{E}-03$ & 1.72 \\
\hline 7 & $2.36 \mathrm{E}-03$ & $5.89 \mathrm{E}-04$ & 0.29 & $2.36 \mathrm{E}-03$ & $8.66 \mathrm{E}-04$ & 0.46 \\
\hline 8 & $7.62 \mathrm{E}-04$ & $3.63 \mathrm{E}-04$ & 0.09 & $1.39 \mathrm{E}-03$ & $1.99 \mathrm{E}-03$ & 0.52 \\
\hline Error reduction $\tilde{P}_{7}$ & & 2.32 & & & 1.90 & \\
\hline Error reduction $\tilde{P}_{8}$ & & 1.63 & & & 0.76 & \\
\hline
\end{tabular}
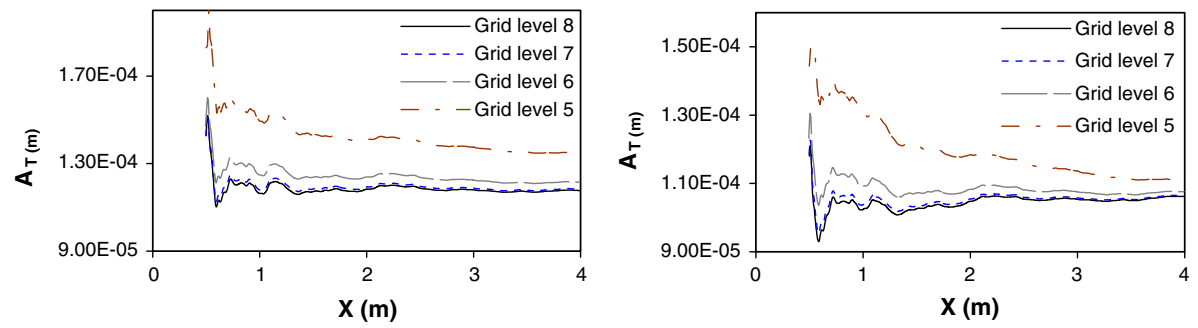

Fig. 4 Average apparent transverse dispersivity, $A_{\mathrm{T}}$, for different grid levels, $j$. Tracer case (left) and brine case (right)

The estimated fraction error, $E_{j}$, for grid discretization level $j$ is defined by

$$
E_{j}=\frac{\left\|\frac{\omega^{j}-\omega^{j-1}}{\omega^{j-1}}\right\|}{r^{\tilde{P}_{j}}-1} r^{\tilde{P}_{j}} .
$$

Roy (2005) showed the importance of the $r^{\tilde{P}_{j}}-1$ factor for obtaining accurate error estimates. Note that this method requires fine grid resolution in the asymptotic range.

Uncertainty in the model is investigated by comparing the differences in salt mass fraction at different grid levels for the whole domain of simulation. Table 3 shows the result of discretization error analysis for both the tracer case and the brine case. Minimum error reductions are obtained for the brine case. For the tracer case, the minimum discretization error is equal to $3.63 \mathrm{E}-04$ at grid level 8 , whereas it is equal to $8.66 \mathrm{E}-04$ for the brine case at grid level 7. Figure 4 shows graphically the effect of different grid levels on the computed apparent dispersivity for both the cases. Note that in this figure, the mass fraction distribution at the beginning of the domain is not considered in the averaging procedure. Obviously, the solutions on grid level 5 for both the cases are not accurate enough and there are noticeable differences compared to the results at finer grid levels. Error reductions for the tracer case and the brine case at $j=7$ are estimated approximately 2 . This test case, hence, confirms that the numerical discretization is second-order consistent in the primary variable $\omega$. Besides, the error fractions are $E_{7}=0.29 \%$ and $E_{7}=0.46 \%$ for the tracer case and brine case, respectively. Moreover, Johannsen et al. (2002) argued that the grid convergence of the numerical scheme depends strongly on the strength of the coupling of flow and transport and on the resolution of the dispersive effects, which is in accordance with our finding.

A similar analysis is conducted on the average mass fraction profiles at the end of the sand box. The corresponding discretization errors are depicted in Fig. 5 for three grid levels on a 
Fig. 5 Spatial (discretization) error of $<\bar{\omega}(\eta, L)>$ for the brine and tracer case

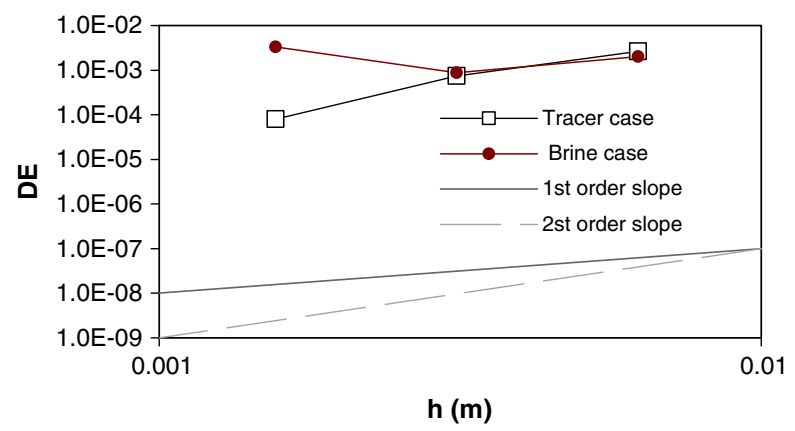

$\log -\log$ scale. Parameter $h$ indicates the grid spacing. The norms decrease in a second-order manner as the grid is refined from grid level 6 to 7 for both cases. Further grid refinement is not needed owing to the fact that the solutions on grid level 7 and 8 concur sufficiently well. Considering computation time, REV concept, and smallness of errors at grid level 7 , we choose a mesh size of $h=0.00312 \mathrm{~m}$ for simulations. We only show one of our analyses here; however, these analyses have been done for different heterogeneities to ensure that all our results have the same order of error.

\subsubsection{Boundary Effects}

The issue is to determine the effects of the boundary conditions on the transverse dispersion flux. In general, it is not trivial to define the boundary condition for experiments to resemble a natural flow system, e.g., an aquifer. Clearly, increasing the height of the tank, $H$, eliminates the effect of the boundaries parallel to the mean flow. Using a relatively large $H$ ensures that boundaries at the top and bottom of the tank have no significant effects on the mixing zone shape. However, left and right boundary conditions would have significant potential to alter the nature of the observed mixing zone.

To investigate the effect of left-hand boundary conditions on the dispersivity coefficients, two boundary conditions are considered, the hydrostatic pressure head or the Constant velocity at the left boundary by using $q=2 \times 10^{-5} \mathrm{~m} / \mathrm{s}, \Delta \rho=200 \mathrm{~kg} / \mathrm{m}^{3}, k=2 \times 10^{-10} \mathrm{~m}^{2}$, and $\sigma_{f}^{2}=0.1,0.5$. Transverse dispersivities are estimated by fitting Eq. 14 to scaled mass fraction distributions results, which are depicted in Fig. 6. The results confirm the significant influence of the different types of boundaries on the dispersive flux. Hydrostatic pressure head condition results in a decrease in the apparent transverse dispersivities in the vicinity of the upstream boundary, which needs a large travel distance to reach the asymptotic values, whereas constant velocity causes a large dispersive flux close to the left boundary. It seems that the latter might have an insignificant impact on the lateral dispersivity coefficients in the system by disregarding the result close to the left BC.

Furthermore, the effect of the variation of right-hand boundary location variation on the current simulation results of the heterogeneous cases is investigated using an extended geometry by using the constant velocity at the left boundary. We, therefore, consider two different sizes of sand box, $L=1,2 \mathrm{~m}$ (See Fig. 6). The influence of downstream boundary conditions on the dispersion coefficients is clearly visible in the presence of strong gravity effects. This effect for weak heterogeneity or smaller gravity number is less pronounced. The error arises due to the nature of boundary conditions used in the right-hand side. Utilizing constant velocity at the end implies no vertical velocity. Moreover, it seems that using the $\mathbf{D} \nabla \omega \cdot \mathbf{n}=0$ condition as outflow boundary is not proper for the brine case. Therefore, it 


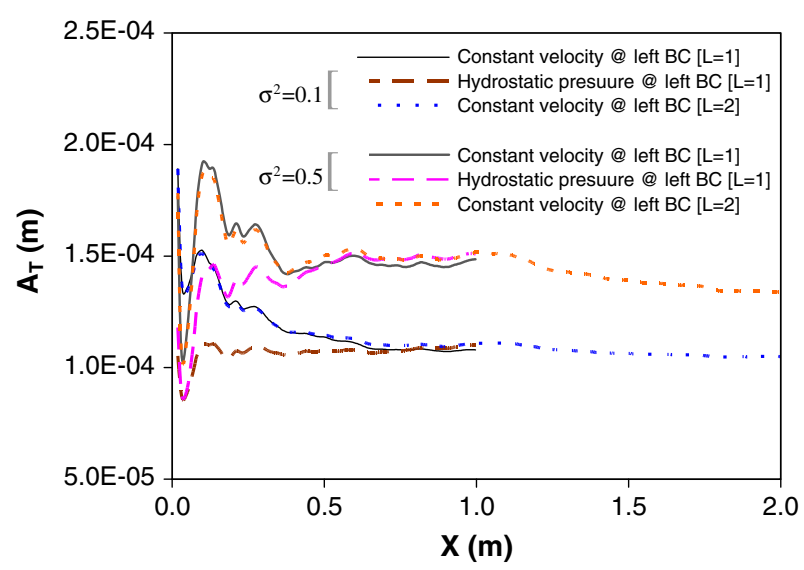

Fig. 6 Boundary condition effects on apparent transverse dispersivity for $N_{\mathrm{g}}=20$. Two geometries with $L=1,2 \mathrm{~m}$ are modeled to investigate the influence of the right boundary condition on apparent dispersivity. Constant flow velocity and hydrostatic pressure boundary conditions are considered

is important to take into account the effect of right $\mathrm{BC}$ on the apparent dispersivity by using the result far from the boundary.

\subsubsection{Convergence of Averaging}

To estimate the lateral apparent dispersivity coefficient for the tracer case and high concentration cases, e.g., brine, several realizations are modeled. First, we take the ensemble average of the mass fractions for each node over $\mathrm{N}$ different realizations by using Eq. 15. Then, Eq. 18 is employed to average the ensemble scaled mass fractions over travel distance, $x$, for each $\eta$ value. Bellin et al. (1992) analyzed dispersion by Monte Carlo simulations using a particle-tracking method. They found that the second-order moments converge after 1000 single realizations for relatively mild heterogeneity. They also showed that more correlation lengths are needed for higher permeability variance. In addition, Tompson and Gelhar (1990) state that spatial averaging over a sufficiently large domain, i.e., sampling enough independent trajectories, mimic ensemble averaging. Thus, averaging over a long tank is employed to study dispersion.

Since we do not know how many realizations are sufficient, we employ two criteria for studying the convergence of averaging procedure. The first criterion, $E_{i}$, represents the convergence of apparent dispersivity, and the second norm, $E E_{i}$, shows the convergence of the ensemble mass fraction profiles.

$$
\begin{gathered}
E_{i}=\left\|\overline{A_{\mathrm{T}}^{i-1}}-\overline{A_{\mathrm{T}}^{i}}\right\|, \\
E E_{i}=\left\|\langle\bar{\omega}(L, \eta)\rangle^{i-1}-\langle\bar{\omega}(L, \eta)\rangle^{i}\right\|,
\end{gathered}
$$

where index $i$ denotes the number of realization used for averaging. Both criteria become small with increasing the number of realizations (Fig. 7). All $E_{i}$ values reach less than $0.1 \%$ of calculated apparent dispersivity. Expectedly, fewer realizations are needed for higher gravity number because of the suppressing effect of gravity force on mass fraction fluctuations. 
Fig. 7 Convergence of ensemble averaging for different gravity numbers $\left(\sigma_{f}^{2}=0.1\right)$. (a) $E_{i}$ (b) $E E_{i}$; (1) $N_{\mathrm{g}}=0$, (2) $N_{\mathrm{g}}=2.5$ (3) $N_{\mathrm{g}}=5$, (4) $N_{\mathrm{g}}=10$

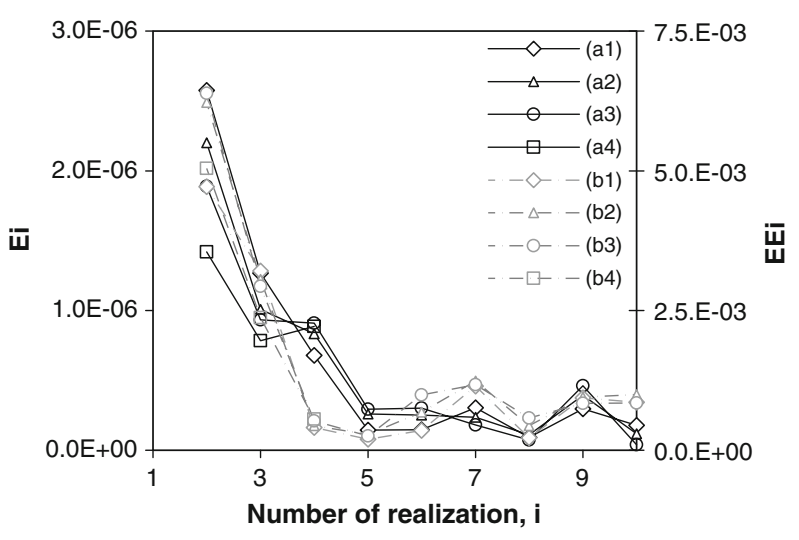

\section{Results and Discussion}

\subsection{Tracer Transverse Dispersivity}

One of the most important issues in complex models is to evaluate the uncertainty of predicted effects. In this study, this uncertainty is caused by the uncertainty in the model input, i.e., different permeability distribution fields. The most widely used method to evaluate the effect of uncertainty on the result is the Monte Carlo method (Dagan 1989). A conservative solute spreads due to the heterogeneities, while it travels in a porous medium encountering the heterogeneity. Thus, we characterize solute distribution in a large scale domain by its macrodispersion coefficients.

Estimating macro scale dispersivity for the tracer case is, thus, essential due to the fact that macro scale transverse dispersivity in the presence of gravity force can only vary in the range between macro scale transverse dispersivity without gravity force, i.e., $A_{\mathrm{T}, \text { tracer }}$, and local scale transverse dispersivity, i.e., $\alpha_{\mathrm{T}}$. Based on the convergence results, we used 20 realizations in our simulations for each heterogeneity distribution to obtain asymptotic macro-dispersivity coefficients. It was found that after 1500 correlation lengths, the second moments, indicating dispersive property, converge (Bellin et al. 1992). In such a case the solute has an opportunity to sample a large range of velocity variations (McLaughlin and Ruan 2001).

Variance values of the average ensemble profiles for different heterogeneity distribution are depicted in Fig. 8. As it can be observed, $\sigma_{\langle\varpi(x)\rangle}^{2}$ reaches an asymptotic value for smaller heterogeneity. It is apparent that fewer realizations are needed for smaller heterogeneity, owing to the decrease of the velocity variations for small heterogeneities compared to highly heterogeneous porous media. Macro-dispersivities listed in Table 4 are used as $A_{\mathrm{T} \text {,tracer }}$ in this study.

\subsection{Density-Affected Apparent Dispersivity}

Owing to the fact that velocity variations induced by heterogeneity cause an asymmetric mixing zone (Fig. 2), in both the tracer case, and brine case the mass fraction distributions are far from the result of homogenous porous media (Figs. 9 and 10). The solute moves slower in lower permeability zones, and moves faster through high permeability zones. The reduction of the mixing zone width is more apparent in Fig. 9, where mass fraction distribution 
Fig. 8 Variance of both (a) a single ensemble profile, $\sigma_{<\omega(x)>}^{2}$, and, (b) the average of ensemble profiles, $\sigma_{<\varpi(x)>}^{2}$ as a function of $\mathrm{x} / \lambda$ for different heterogeneities; (1) $\sigma_{f}^{2}=0.05$, (2) $\sigma_{f}^{2}=0.1$ (3) $\sigma_{f}^{2}=0.25$, and (4) $\sigma_{f}^{2}=0.5$

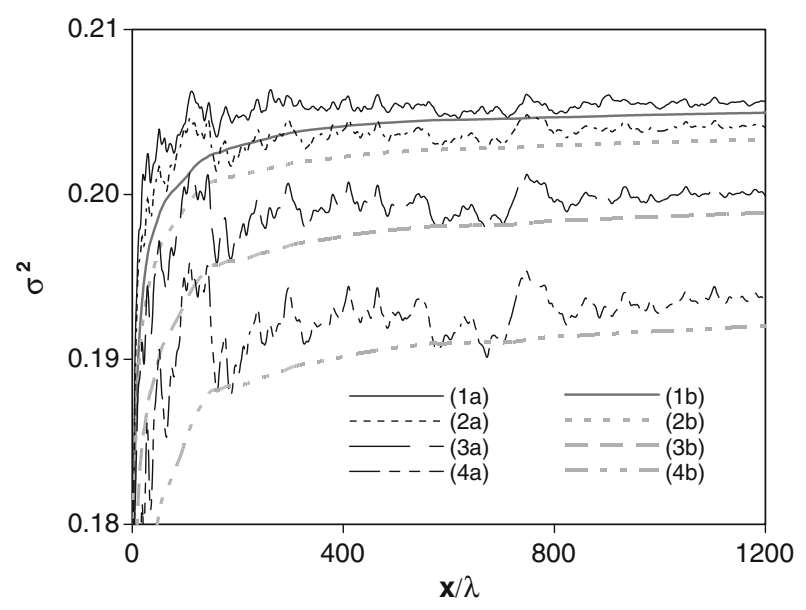

\begin{tabular}{lll}
\hline Heterogeneity & $A_{\mathrm{T}, \text { tracer }} \times 10^{-4}$ & $\left(A_{\mathrm{T}, \text { tracer }}-\alpha_{\mathrm{T}}\right) / \alpha_{\mathrm{T}}(\%)$ \\
\hline$\sigma_{f}^{2}=0.05$ & 1.11 & 11 \\
$\sigma_{f}^{2}=0.1$ & 1.20 & 20 \\
$\sigma_{f}^{2}=0.25$ & 1.47 & 47 \\
$\sigma_{f}^{2}=0.5$ & 1.89 & 89 \\
\hline
\end{tabular}

Table 4 Transverse macro-dispersivity for different heterogeneous porous media

in a weakly heterogeneous porous medium for both the tracer and brine cases are illustrated. The mixing zone width decreases in the brine case, although the mass fraction fluctuation pattern in the brine case mimics the same pattern in the tracer case (Fig. 10). This is due to the fact that we use the same permeability distribution for both the cases. In this figure, fluctuations of local mass fraction for five different $\eta$ values, i.e., iso-mass-fraction lines in homogenous case, against travel distance, $x$, are plotted. In homogenous porous media, these curves are horizontal in the new coordinates $[x, \eta]$. Hence, the results of the brine case are closer to the homogenous case due to the fact that there are less velocity variations. This confirms the hypothesis that gravity is responsible for a stabilizing effect resulting from spatial heterogeneities. These results, hence, show the interaction between gravity forces and convective forces so that gravity flow cause a rotation in the opposite direction of rotation induced by heterogeneity at the interface between a lighter and heavier fluid toward a stable situation (Schotting and Landman 2004). The variance of the average of ensemble profiles, i.e., $\sigma_{<\varpi(x)>}^{2}$, calculated for different gravity numbers are plotted in Fig. 11, which shows the reducing effect of gravity on the solute spreading transversally. These confirm the observation from laboratory experiments, such as Grane and Gardner (1961) and Starke and Koch (2006), and the analytical study of Thiele (1997).

To explore the stabilizing effect of gravity on the dispersive mass flux, we perform five series of numerical experiments:

- Constant flow rate $\left(q=2 \times 10^{-5} \mathrm{~m} / \mathrm{s}\right)$ but variable density contrasts $\left(\Delta \rho=0-200 \mathrm{~kg} / \mathrm{m}^{3}\right)$ for different heterogeneities $\left(\sigma_{f}^{2}=0.1,0.25,0.5\right)$. 
Fig. 9 Computed scaled mass fraction counters for the tracer and brine case for one single realization $\left(k=2 \times 10^{-10} \mathrm{~m}^{2}\right.$, $\left.\sigma_{f}^{2}=0.1\right)$. The wiggles are less in brine case due to the stabilizing effect of gravity

Fig. 10 Local scaled mass fraction for different $\eta$ values versus length of tank for one realization $\left(k=2 \times 10^{-10} \mathrm{~m}^{2}\right.$, $\left.\sigma_{f}^{2}=0.1, N_{\mathrm{g}}=20\right)$. Solid lines indicate the tracer case; dashed lines indicate brine case
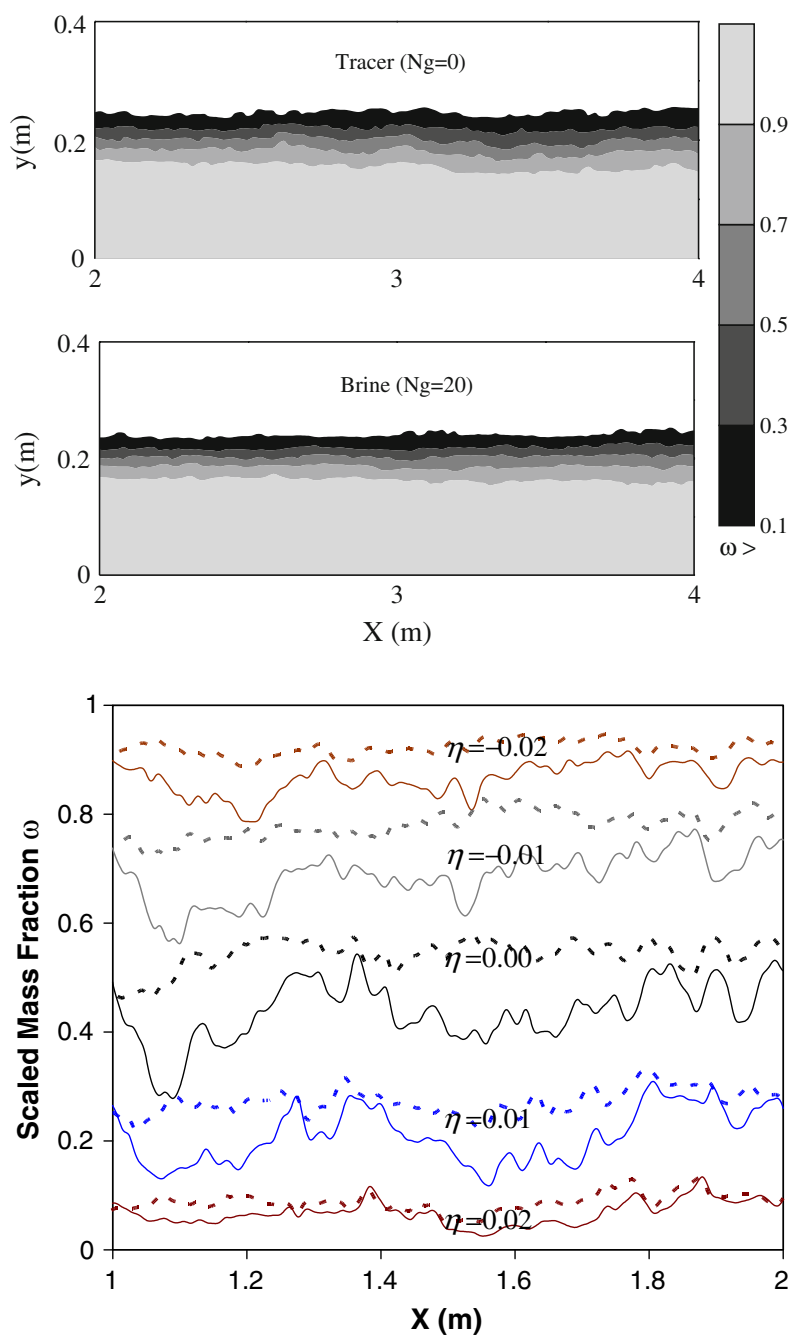

- Constant density contrast $\left(\Delta \rho=200 \mathrm{~kg} / \mathrm{m}^{3}\right)$ but variable flow rate $\left(q=2 \times 10^{-5}\right.$ $\left.-1 \times 10^{-4} \mathrm{~m} / \mathrm{s}\right)$ with moderate heterogeneities $\left(\sigma_{f}^{2}=0.5\right)$ for different average permeabilities $\left(k=2 \times 10^{-10}, 4 \times 10^{-10} \mathrm{~m}^{2}\right)$.

For each simulation, we estimate the apparent dispersivity from the simulation results using Eq. 14. We use it in order to characterize the effect of gravity force on dispersive flux for different configurations. We illustrate the scaled apparent transverse dispersivities from numerical simulations against the gravity number in Fig. 12.

In addition, a series of simulations are conducted for different velocities in the case of brine, i.e., $\Delta \rho=200 \mathrm{Kg} / \mathrm{m}^{3}$. Scaled dispersions, i.e., $D_{\mathrm{T}} / D_{\mathrm{m}}$, are plotted against transverse Peclet number in Fig. 13. Transverse Peclet number is given by $P e_{\mathrm{T}}=q \alpha_{\mathrm{T}} / n D_{\text {eff }}$. Figure 13 shows that in the case of coarse sand, e.g., $k=2 \times 10^{-10} \mathrm{~m}^{2}$, the effect of density gradient on transverse dispersivity is more pronounced when molecular diffusion and mechanical dispersion are of a similar order of magnitude. Therefore, it is clear that for smaller permeability, 
Fig. 11 Variance of the average of ensemble profiles, $\sigma_{<\varpi(x)>}^{2}$, at each travel distance for different gravity numbers: (1) $N_{\mathrm{g}}=0$, (2) $N_{\mathrm{g}}=2.5$ (3) $N_{\mathrm{g}}=5$, and (4) $N_{\mathrm{g}}=10$

Fig. 12 Scaled transverse dispersivity against gravity number for different heterogeneities. Constant flow rate $\left(q=2 \times 10^{-5}\right)$ but variable densities, (1) $\sigma_{f}^{2}=0.1$,

(2) $\sigma_{f}^{2}=0.25$, and (3) $\sigma_{f}^{2}=0.5$; Constant density contrast and heterogeneity

$\left(\Delta \rho=200, \sigma_{f}^{2}=0.5\right)$ but variable flow rate

(4) $k=2 \times 10^{-10} \mathrm{~m}^{2}$

(5) $k=4 \times 10^{-10} \mathrm{~m}^{2}$
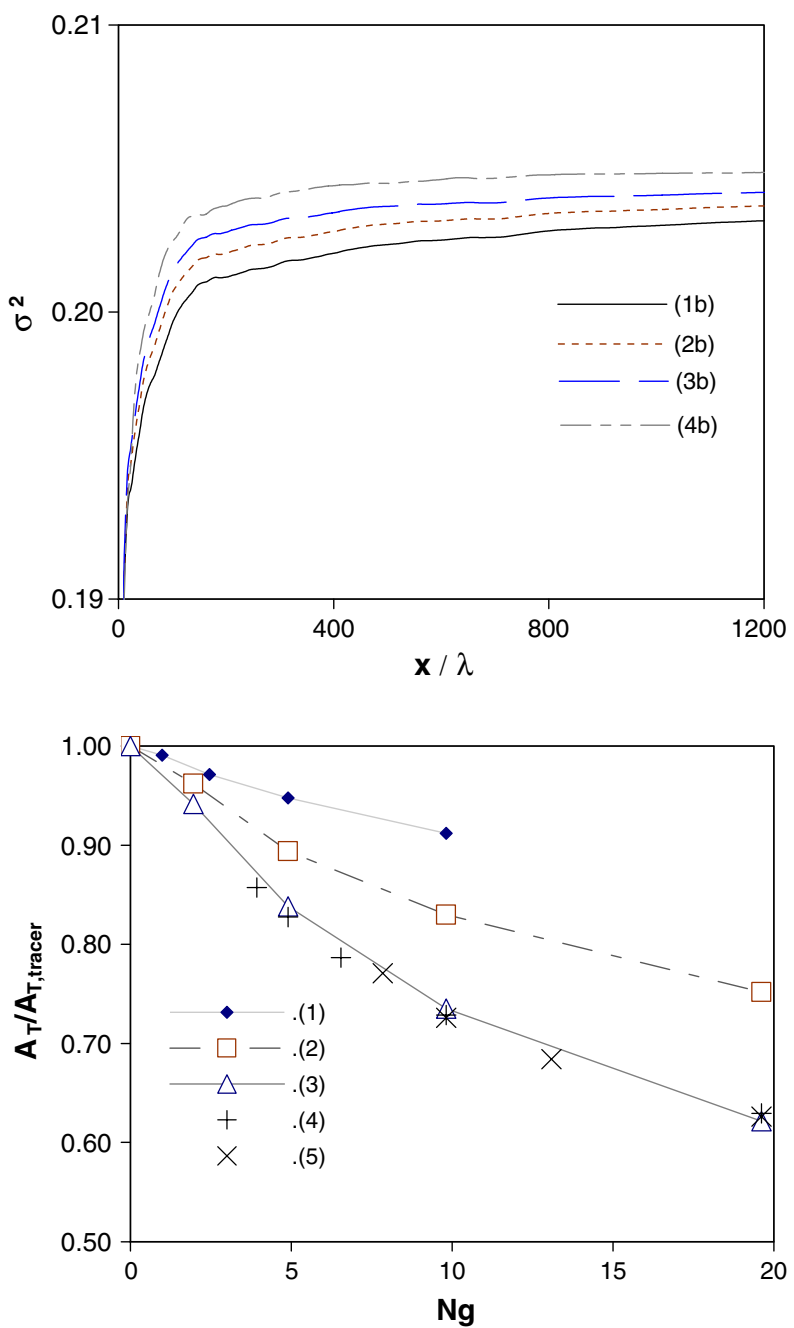

the effect of gravity on dispersion becomes less pronounced due to the fact that the flow is diffusion dominated which was also observed by Grane and Gardner (1961). A nonlinear dispersion equation, $D=D_{\mathrm{m}}+A_{\mathrm{T}} q^{\mathrm{m}}$, used by Watson et al. (2002b) is fitted to the results of the brine case. For the brine case, $m$ is equal to 1.04, which shows a small deviation in the dispersion of the brine case from the dispersion of the tracer case where $\mathrm{m}$ is equal to 1. Watson et al. (2002b) obtained a higher value for the case of longitudinal dispersion in a similar porous medium, e.g., $m=1.44$.

In the case of longitudinal dispersion, Landman et al. (2007a) estimated a one order of magnitude reduction in the scaled macro-dispersivity for a relatively small gravity number, e.g., $N_{\mathrm{g}}=1.6$. In the case of transverse dispersion for the same porous medium properties, this reduction is limited to $20 \%$ for a high gravity number, e.g., $N_{\mathrm{g}}=16$. In other words, the reduction in the scaled transverse macro-dispersivity for the gravity number Landman (2005) used, i.e., $N_{\mathrm{g}}=1.6$, is limited to $2 \%$. This is due to the less pronounced effect of heterogeneity on the increase of dispersion coefficient in the transverse direction, as compared 
Fig. 13 Scaled transverse dispersion against transverse Peclet number $\left(k=2 \times 10^{-10} \mathrm{~m}^{2}\right)$

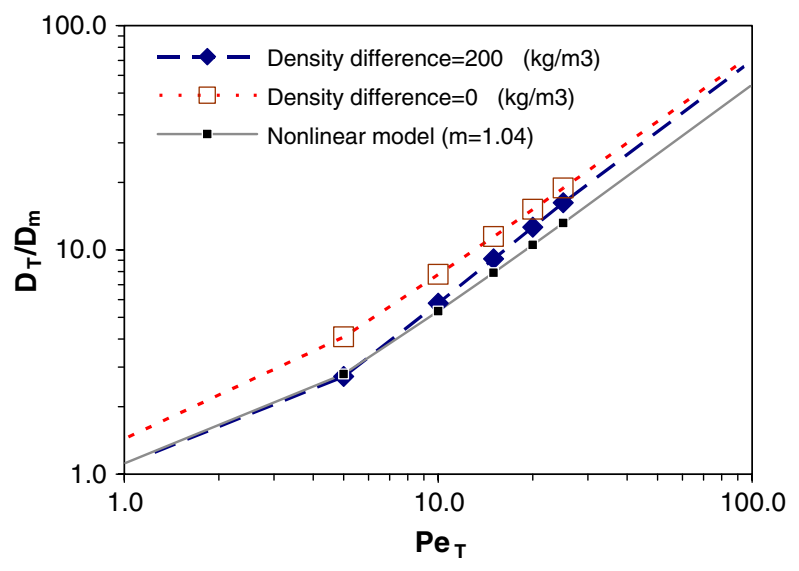

to longitudinal dispersion coefficient, owing to the fact that transverse velocity components are smaller than those in the longitudinal direction, i.e., parallel to the main flow direction. It is also stated by Dagan (1988) and Cirpka and Attinger (2003) that in contrast to longitudinal macro-dispersivity, transverse macro-dispersivity approaches an asymptotic value only slightly larger than the local scale coefficient. Hence, this reduction is limited to this small range, while the maximum reduction is equal to $\left(A_{\mathrm{T}, \text { tracer }}-\alpha_{\mathrm{T}}\right)$. We can observe three important features in Figs. 12 and 13:

- The difference in the trend of reduction for different heterogeneities originates from the difference in the corresponding medium configurations. It implies that for a stronger heterogeneous porous medium, gravity forces have more possibility to diminish vertical advection induced by the heterogeneities. In particular, this is true when the main flow direction is perpendicular to the gravity direction. As illustrated by Landman et al. (2007b), the increase in heterogeneity acts in an opposite manner.

- The effect of gravity on transverse dispersion, i.e., $D_{\mathrm{T}}$, is more pronounced when $P e_{\mathrm{T}}$ and $N_{\mathrm{g}}$ are large.

- While gravitational forces come into play, the dispersion flux cannot be explained accurately by linear Fick's law at the macro scale. In that case, dispersion becomes a function of density contrast or gravity number as well as effective diffusion, macro-dispersivity, and velocity.

\section{The Theory of Hassanizadeh and Leijnse}

Hassanizadeh and Leijnse (1995) suggested a nonlinear extension of Fick's law which includes an additional parameter, $\beta$.

$$
(1+\beta|\mathbf{J}|) \mathbf{J}=-\rho \mathbf{D} \cdot \nabla \omega
$$

The second term in the dispersive mass flux, i.e., $\beta|\mathbf{J}| \mathbf{J}$, is considered to represent the resistance of the system to dispersion due to the mass fraction gradients. Hassanizadeh and Leijnse stated that the parameter $\beta$ is independent of the fluid properties and might be a function of the flow velocity.

Since no experimental data in a two-dimensional flow domain are available, we rely on numerical experiments to assess the theory of Hassanizadeh and Leijnse. 


\subsection{Model Description}

We assume a uniform and constant flow field for a two-dimensional domain, i.e., $q_{y}=0$ and $q_{x}=q \quad \forall(x, y) \in \Re$. In that case, the governing equation in an effectively homogeneous porous medium for steady state is given by

$$
q \frac{\partial \rho \omega}{\partial x}=-\left(\frac{\partial}{\partial x}\left(J_{x}\right)+\frac{\partial}{\partial y}\left(J_{y}\right)\right)
$$

Equation 26 for a two-dimensional domain yields

$$
\begin{aligned}
& \left(1+\beta \sqrt{\left(J_{x}\right)^{2}+\left(J_{y}\right)^{2}}\right) J_{x}=-\rho D_{x x} \frac{\partial \omega}{\partial x} \\
& \left(1+\beta \sqrt{\left(J_{x}\right)^{2}+\left(J_{y}\right)^{2}}\right) J_{y}=-\rho D_{y y} \frac{\partial \omega}{\partial y}
\end{aligned}
$$

where

$$
\begin{aligned}
& D_{x x}=D_{\text {eff }}+\alpha_{L} q \\
& D_{y y}=D_{\text {eff }}+\alpha_{\mathrm{T}} q
\end{aligned}
$$

Writing dispersive mass fluxes in $x$ and $y$ directions, i.e., $J_{x}$ and $J_{y}$, and assuming $J_{y}>0$, i.e., stable displacement, by virtue of isotropic $\beta$ yields

$$
\begin{aligned}
& J_{x}=-D_{x x} \frac{\partial \omega}{\partial x}\left(\frac{-1+\sqrt{1+4 \beta \rho q \sqrt{\left(D_{x x} \frac{\partial \omega}{\partial x}\right)^{2}+\left(D_{y y} \frac{\partial \omega}{\partial y}\right)^{2}}}}{2 \beta \sqrt{\left(D_{x x} \frac{\partial \omega}{\partial x}\right)^{2}+\left(D_{y y} \frac{\partial \omega}{\partial y}\right)^{2}}}\right) \\
& J_{y}=-D_{y y} \frac{\partial \omega}{\partial y}\left(\frac{-1+\sqrt{1+4 \beta \rho q \sqrt{\left(D_{x x} \frac{\partial \omega}{\partial x}\right)^{2}+\left(D_{y y} \frac{\partial \omega}{\partial y}\right)^{2}}}}{2 \beta \sqrt{\left(D_{x x} \frac{\partial \omega}{\partial x}\right)^{2}+\left(D_{y y} \frac{\partial \omega}{\partial y}\right)^{2}}}\right)
\end{aligned}
$$

The solutions of $J_{x}$ and $J_{y}$ with an anisotropic $\beta$ parameter are more complicated. Next, we assume that the dispersive mass flux gradient in the $x$-direction is small and negligible compared to that in the $y$-direction, i.e., $\partial J_{y} / \partial y>>\partial J_{x} / \partial x \approx 0$. Under this assumption, after scaling $\omega$ according to $\hat{\omega}=\omega / \omega_{s}$, Eqs. 27 and 32 reduce to

$$
\begin{aligned}
q \frac{\partial \hat{\omega}}{\partial x} & =-\frac{1}{\omega_{s} \rho_{f} \mathrm{e}^{\gamma \rho \omega_{s} \hat{\omega}}\left(1+\hat{\omega} \omega_{s} \gamma_{\rho}\right)} \frac{\partial}{\partial y}\left(J_{y}\right) \\
J_{y} & =\frac{-1+\sqrt{1-4 \beta \omega_{s} \rho_{f} \mathrm{e}^{\gamma_{\rho} \omega_{s} \hat{\omega}} D_{y y} \frac{\partial \hat{\omega}}{\partial y}}}{2 \beta}
\end{aligned}
$$

This set of equations is solved numerically using a standard five-point stencil finite difference scheme. The Jacobian matrix is solved using a Newton-Raphson method. Moreover, initial conditions are introduced using Eq. 12 with the apparent transverse dispersivity for the tracer 

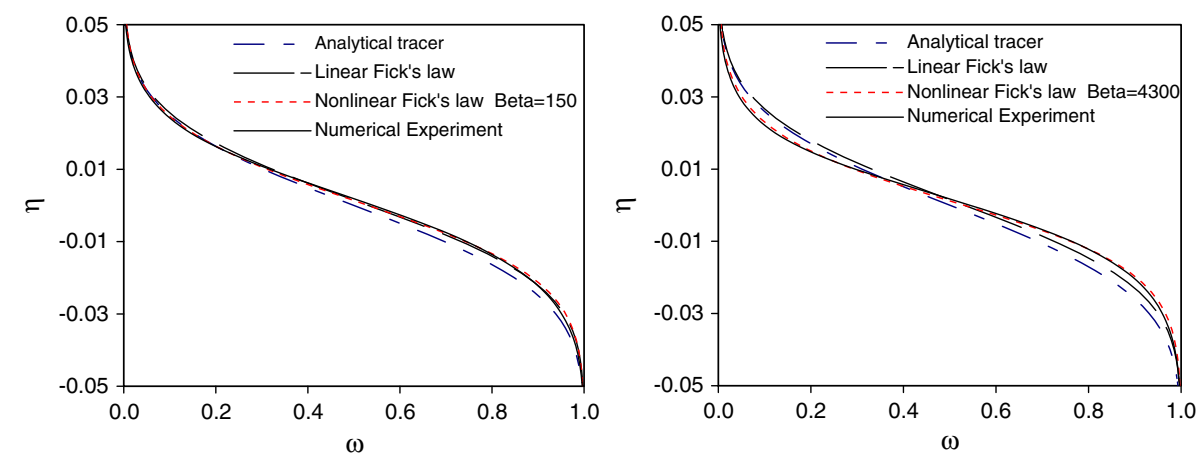

Fig. 14 Scaled mass fraction profiles for $\sigma^{2}=0.5, \Delta \rho=200 \mathrm{~kg} / \mathrm{m}^{3}$, and $q=10^{-4}($ left $) ; q=2 \times 10^{-5} \mathrm{~m} / \mathrm{s}$ (right)

case. Furthermore, the boundary conditions are similar to the boundary conditions used in the numerical $d^{3} f$ experiment.

In a stable displacement, obviously, the mass fraction gradient in the vertical direction is always negative. We calculate profiles in terms of scaled mass fraction $\hat{\omega}$ and fit the $\beta$ parameter by matching the maximum gradient of the calculated profiles of the Hassanizadeh's model and those obtained with $d^{3} f$, to which we refer as "experimental result."

\subsection{Results}

The nonlinear model of Hassanizadeh is solved under two simplifying assumptions. First, assuming a constant flow rate in the flow domain by disregarding the fluid mass balance equation, second, neglecting $\partial J_{x} / \partial x$ in comparison with $\partial J_{y} / \partial y$.

In order to study the validity and applicability of this nonlinear model, a series of simulations is conducted. Simulations include different mean specific discharge, density differences, and the log-permeability variances. We used a fitting procedure to determine the $\beta$ parameter to examine the dependency of $\beta$ on $\Delta \rho, q$, and $\sigma_{f}^{2}$.

The unknown $\beta$ parameter in Hassanizadeh's model is fitted to the numerical experiments for a range of velocities $\left(2 \times 10^{-5}\right.$ to $\left.1 \times 10^{-4} \mathrm{~m} / \mathrm{s}\right)$ with a fixed density contrast $\left(\Delta \rho=200 \mathrm{~kg} / \mathrm{m}^{3}\right)$ and a fixed $\log$ permeability variance $\left(\sigma_{f}^{2}=0.5\right)$. In Fig. 14 , the ensemble averages of numerical experiments are compared with the linear model (Eq. 4), the analytical solution (Eq. 14), and the nonlinear model of Hassanizadeh (Eq. 35). It is shown that for a higher flow velocity, the result of both linear and nonlinear models are close to the result of the numerical experiments. This also confirms that at relatively low gravity number, $N_{\mathrm{g}}=4$, the linear form of Fick's law is valid in weakly heterogeneous porous media, whereas at lower flow velocity, i.e., higher gravity number, the linear model cannot predict the numerical experiment result correctly. However, the nonlinear model of Hassanizadeh is in good agreement. Figure 15 clearly shows the differences between these model results by using the scaled mass fraction gradient. It should be noted that as a result of disregarding the fluid mass balance equation in the nonlinear model of Hassanizadeh, a slight shift can be observed, which is due to the volume effect. Nevertheless, it does not alter the shape of the mass fraction distribution.

The model of Hassanizadeh and Leijnse has been tested against various laboratory and numerical experiments for longitudinal dispersivity only. In Fig. 16 (Left), the flow rate dependence of $\beta$ is depicted for a porous medium with $\sigma_{f}^{2}=0.5$ in the case of transverse 

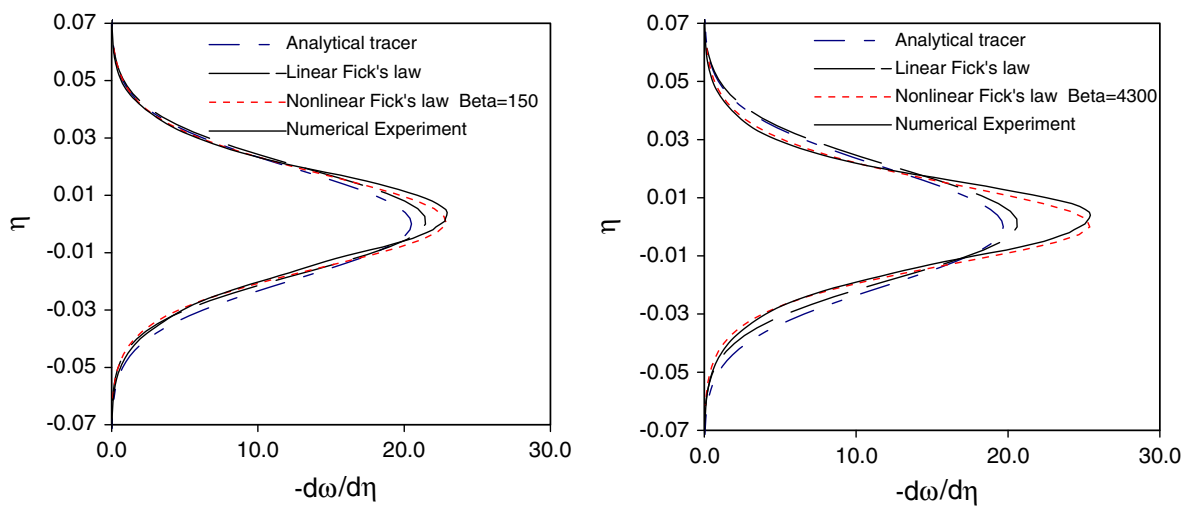

Fig. 15 Scaled mass fraction gradients for $\sigma^{2}=0.5, \Delta \rho=200 \mathrm{~kg} / \mathrm{m}^{3}$, and $q=10^{-4}($ left $) ; q=2 \times 10^{-5} \mathrm{~m} / \mathrm{s}$ (right)
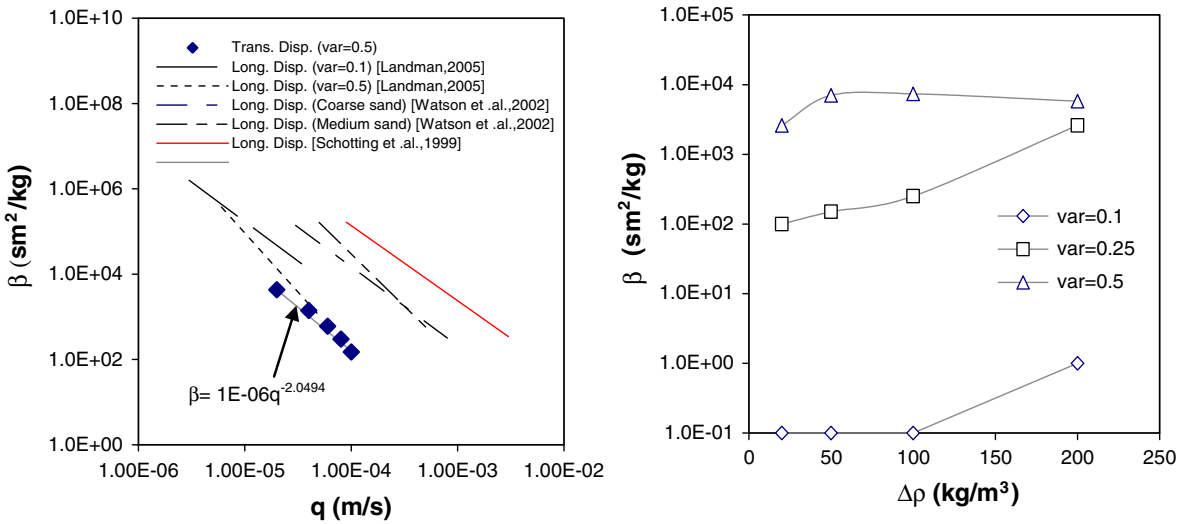

Fig. 16 Dependence of $\beta$ on the flow rate (left), and on the density contrast and heterogeneity (right)

dispersivity $\left(\Delta \rho=200 \mathrm{~kg} / \mathrm{m}^{3}\right)$; also, other functions suggested for longitudinal dispersivity are shown in this figure (Landman 2005; Watson et al. 2002b; Schotting et al. 1999). Obviously, each of them has a different behavior. It is clear that the parameter $\beta$ for longitudinal dispersivity and for transverse dispersivity is inversely proportional to the flow rate, and on a $\log -\log$ scale, it decreases linearly with flow rate. Note that almost all lines in this figure are parallel.

In addition, the parameter $\beta$ is fitted to the numerical experiments for different density contrasts and different $\log$-permeability variances $\left(\sigma_{f}^{2}=0.1,0.25,0.5\right)$ at a constant specific discharge of $2 \times 10^{-5} \mathrm{~m} / \mathrm{s}$, see Fig. 16 (Right). In all cases, the $\beta$ values are increasing with $\Delta \rho$ up to one order of magnitude. In weakly heterogeneous porous media, i.e., $\sigma_{f}^{2}=0.1,0.25$, the $\beta$ values remain constant for density contrast less than $100 \mathrm{~kg} / \mathrm{m}^{3}$ and increases for a higher density contrast, i.e., $\Delta \rho>100 \mathrm{~kg} / \mathrm{m}^{3}$. Whereas for more pronounced heterogeneity, i.e., $\sigma_{f}^{2}=0.5$, the $\beta$ values are almost constant for $\Delta \rho>50 \mathrm{~kg} / \mathrm{m}^{3}$. The parameter $\beta$ for small $\log$-permeability variance $\left(\sigma_{f}^{2}=0.1\right)$ is small, which is due to the small effect of gravity on transverse dispersion for such a weak heterogeneity, thus the linear form of Fick's law is still appropriate for this situation. Furthermore, it can be observed that the more 
Table 5 Dependence of $\beta$ on different parameters

\begin{tabular}{lllll}
\hline & $\begin{array}{l}\text { Longitudinal } \\
\text { dispersivity } \\
\text { (Schotting et al. 1999) }\end{array}$ & $\begin{array}{l}\text { Longitudinal } \\
\text { dispersivity } \\
\text { (Watson et al. 2002b) }\end{array}$ & $\begin{array}{l}\text { Longitudinal } \\
\text { dispersivity } \\
\text { (Landman 2005) }\end{array}$ & $\begin{array}{l}\text { Transverse } \\
\text { dispersivity }\end{array}$ \\
Velocity dependent & $\beta=f(q)$ & $\beta=f(q)$ & $\beta=f(q)$ & $\beta=f(q)$ \\
Time dependent & & & $\beta=f(t)$ & $\beta=f(\Delta \rho)$ \\
Density difference & & & $\beta=f(\Delta \rho)$ & $\beta=f\left(\sigma^{2}\right)$ \\
Permeability variance & & $\beta=f\left(\sigma^{2}\right)$ & $\beta \neq \beta_{y}$ \\
Direction dependence & & & $\beta_{x}$ \\
\hline
\end{tabular}

heterogeneous porous medium, the higher the $\beta$ values are. This has been confirmed for longitudinal dispersivity by Landman et al. (2007a) and Watson et al. (2002a).

Considering the $\beta$ values reported by Landman (2005) and these values for porous media with $\sigma_{f}^{2}=0.1$, the nonlinear parameter of Hassanizadeh's model is a tensor, i.e., different values for each direction. This is in accordance with the assumption that the $\beta$ parameter is anisotropic.

By virtue of Eq. 26, it can be observed that the higher the $\beta$ parameter the smaller the magnitude of $J$. Therefore, disregarding $\partial J_{x} / \partial x$ can be justified by

- Having smaller mass fraction gradient in the $x$ direction $(\partial \omega / \partial x)$ than that in the $y$ direction $(\partial \omega / \partial y)$.

- Knowing that $\beta_{x}$ is higher than $\beta_{y}$, which causes $J_{x}$ to be much smaller than $J_{y}$.

Based on our analysis, important aspects of this model are:

- This nonlinear model is able to predict the experimental results in the density-dependent flow problem by knowing the $\beta$ parameter. In spite of that, the linear form of Fick's law is still valid for nearly homogeneous porous media $\left(\sigma_{f}^{2}=0.1\right)$ due to the small effect of the density contrast on dispersive flux.

- Considering the $\beta$ values obtained for transverse dispersivity and those calculated by Landman (2005) shows that the $\beta$ in direction parallel to the flow direction is much higher than that in a direction perpendicular to the main flow direction. This is in accordance with assuming $\partial J_{y} / \partial y>>\partial J_{x} / \partial x \approx 0$.

- Regarding our finding and other studies, the $\beta$ parameter depends on several factors, such as flow velocity, travel time, density contrast, heterogeneity, and direction. Table 5 illustrates these dependencies for a number of studies.

\section{Conclusions}

This paper reports numerical and theoretical results of the transverse dispersion coefficient in heterogeneous porous media of stable case. The following conclusion arising from this analysis can be drawn:

- Stabilizing gravity forces are responsible for the reduction of the mixing zone width at steady state. The gravity number, hence, appears to be the controlling parameter for dispersive flux. In the case of a large gravity number, the local fluctuations in mass fraction decrease due to gravity-driven rotational flow. Scaled mass fraction distributions, therefore, tend to reach the same result as in the homogeneous case. The decrease of 
transverse dispersivity with gravity number is in accordance with laboratory experiments of Starke (2005).

- The effect of density on solute macro-dispersion is not included in the classical, linear, formulation of Fick's law. Owing to the fact that dispersivity is a function of the gravity number, i.e., dispersivity is not only a medium property.

- The effects of density-driven flow on dispersive flux in a porous medium with very low permeability variations is smaller than in the case of relatively higher permeability variations.

- The reduction of the transverse dispersivity due to density gradients is much less than that of the longitudinal dispersivity for the same porous medium. In addition, for media with larger permeability variations, the effects of high density gradients on dispersive mass flux are more pronounced.

- Computation error studies ensure that the numerical uncertainties in the results are small. However, refined grids are required to obtain solutions with an acceptable level of accuracy. The produced profiles, hence, are accurate for comparison with the nonlinear dispersive models.

- Mass fraction averages are taken over a line in 2-D, rather than a surface in 3-D, which might increase the uncertainty in the averaged dispersivity. Using several realizations in this study and the ensemble average of mass fraction to calculate the transverse dispersion coefficient is similar to simulation with three-dimensional models for the case where a rectangular plane source is used at the left boundary.

- Estimating smaller $\beta$ values, which is the fitting parameter of Hassanizadeh's model, for the transverse dispersivity in comparison to that for the longitudinal dispersivity proves that the $\beta$ parameter is not isotropic.

- While the main cause of the nonlinear behavior of dispersion is the interaction between density contrast and vertical velocity, which is a product of average velocity and heterogeneity, accounting for it explicitly in the macro-scale model is essential.

Acknowledgements We wish to thank Majid Hassanizadeh, Rainer Helmig, and Robert Zimmerman for their scientific feedback and comments. We thank IWR in Heidelberg for using their cluster.

Open Access This article is distributed under the terms of the Creative Commons Attribution Noncommercial License which permits any noncommercial use, distribution, and reproduction in any medium, provided the original author(s) and source are credited.

\section{References}

Bastian, P. et al.: UG-a flexible software toolbox for solving partial differential equations. Comput. Visual. Sci. 1, 27-40 (1997)

Bellin, A., Salandin, P., Rinaldo, A.: Simulation of dispersion in heterogeneous porous formations: statistics, first-order theories, convergence of computations. Water Resour. Res. 28(9), 2211-2227 (1992)

Burr, D.T., Sudicky, E.A., Na, R.L.: Nonreactive and reactive solute transport in three-dimensional heterogeneous porous media: mean displacement, plume spreading, and uncertainty. Water Resour. Res. 30(3), 791-815 (1994)

Chaudhuri, A., Sekhar, M.: Stochastic modeling of solute transport in 3-D heterogeneous porous media with random source condition. Stoch. Environ. Res. Risk Assess. 21, 159-173 (2006)

Cirpka, O.A., Attinger, S.: Effective dispersion in heterogeneous media under random transient flow conditions. Water Resour. Res. 39(9), 1257 (2003)

Dagan, G.: Time-dependent macrodispersion for solute transport in anisotropic heterogeneous aquifers. Water Resour. Res. 24(9), 1491-1500 (1988)

Dagan, G.: Flow and Transport in Porous Formations. Springer, Berlin (1989) 
Diersch, H.-J., Kolditz, O.: variable-density flow and transport in porous media: approaches and challenges. Adv. Water Resour. 25(8-12), 899-944 (2002)

Egorov, A.G., Demidov, D.E., Schotting, R.J.: On the interaction between gravity forces and dispersive brine fronts in micro-heterogeneous porous media. Adv. Water Resour. 28(1), 55-68 (2005)

Fein, E.: $d^{3} f$-ein Programmpaket zur Modellierung von Dichtestromungen. Tech. Rep. GRS-139, GRS, Braunschweig, Germany (1998)

Frolkovic, P.: Consistent velocity approximation for density driven flow and transport. In: Advanced Computational Methods in Engineering, pp. 603-611. Shakeer Publishing, Maastrict (1998)

Gelhar, L.W., Axness, C.L.: Three dimensional stochastic analysis of macrodispersion in aquifers. Water Resour. Res. 19(1), 161-180 (1983)

Gelhar, L.W.: Stochastic subsurface hydrology from theory to applications. Water Resour. Res. 22, 1355-1453 (1986)

Grane, F.E., Gardner, G.H.F.: Measurements of transverse dispersion in granular media. J. Chem. Eng. Data 6(2), 283-287 (1961)

Ham, P.A.S., Schotting, R.J., Prommer, H., Davis, G.B.: Effects of hydrodynamic dispersion on plume lengths for instantaneous biomolecular reactions. Adv. Water Resour. Res. 27, 803-813 (2004)

Hassanizadeh, S.M.: Derivation of basic equations of mass transport in porous media. Part 2. Generalized Darcy's and Fick's laws. Adv. Water Resour. 9, 207-222 (1986)

Hassanizadeh, S.M.: Experimental study of coupled flow and mass transport: a model validation exercise. In: Calibration and reliability in groundwater modelling, Proceedings of the Model Care Conference, IAHS publication No. 195, pp 241-250 (1990)

Hassanizadeh, S.M., Leijnse, A.: On the modeling of brine transport in porous media. Water Resour. Res. 24(3), 321-330 (1988)

Hassanizadeh, S.M., Leijnse, A.: A non-linear theory of high-concentration gradient dispersion in porous media. Adv. Water Resour. 18(4), 203-215 (1995)

Hassanizadeh, S.M., Leijnse, A., de Vries, W.J., Stapper, R.A.M.: Experimental study of brine transport in porous media. RIVM Report 728514005, Bilthoven, The Netherlands (1990)

Jiao, C.-Y., Hotzl, H.: An experimental study of miscible displacements in porous media with variation of fluid density and viscosity. Transp. Porous Media 54, 125-144 (2004)

Johannsen, K.: On the validity of the Boussinesq approximation for the Elder problem. Computat. Geosci. 7(3), 169-182 (2003)

Johannsen, K.: Numerische Aspekte dichtegetriebener Stroemung in poroesen Medien. Heidelberg University, Habilitationsschrift (2004)

Johannsen, K., Kinzelbach, W., Oswald, S., Wittum, G.: The saltpool benchmark problem—numerical simulation of saltwater upconing in a porous medium. Adv. Water Resour. 25(3), 335-345 (2002)

Johannsen, K., Oswald, S., Held, R., Kinzelbach, W.: Numerical simulation of three-dimensional saltwaterfreshwater fingering instabilities observed in a porous medium. Adv. Water Resour. 29(11), 1690-1704 (2006)

Landman, A. J.: Analysis of physical mechanisms underlying density-dependent transport in porous media. Utrecht University (2005)

Landman, A.J., Johannsen, K., Schotting, R.J.: Density-dependent dispersion in heterogeneous porous media. Part I: A numerical study. Water Resour. Res. 30(12), 2467-2480 (2007a)

Landman, A.J., Schotting, R.J., Egorov, A., Demidov, D.: Density-dependent dispersion in heterogeneous porous media Part II: Comparison with nonlinear models. Water Resour. Res. 30(12), 2481-2498 (2007b)

Leroy, C., Hulin, J.P., Lenormand, R.: Tracer dispersion in stratified porous media: influence of transverse dispersion and gravity. J. Contaminant Hydrol. 11, 51-68 (1992)

McLaughlin, D., Ruan, F.: Macrodispersivity and large-scale hydrogeologic variability. Transp. Porous Media 42(1-2), 133-154 (2001)

Moser, H.: Einfluß der Salzkonzentration auf die hydrodynamische Dispersion im porösen Medium. Mitteilungen des Institutes für Wasserbau u. Wasserwirtschaft. Mitteilung nr. 128, TU Berlin,

Oberkampf, W.L., Trucano, T.G.: Verification and validation in computational fluid dynamics. Prog. Aerospace Sci. 38, 209-272 (2002)

Pickens, J.F., Grisak, G.E.: Scale-dependent dispersion in a stratified granular aquifer. Water Resour. Res. 17(4), 1191-1211 (1981)

Robin, M.J.L., Gutjar, A.L., Sudicky, E.A., Wilson, J.L.: Cross-correlated random field generation with the direct Fourier transform method. Water Resour. Res. 29(7), 2385-2397 (1993)

Roy, C.J.: Review of code and solution verification procedures for computational simulation. J. Comput. Phys. 205(1), 131-156 (2005)

Schotting, R., Landman, A.L.: Towards a physically based theory of high-concentration-gradient dispersion in porous media. Experimental, theoretical and numerical studies. In: Ingham, D.B., et al. (eds.) Emerging 
Technologies and Techniques in Porous Media, NATO Science Series II, vol. 134, pp. 321-336. Kluwer Academic. Publishers, Dordrecht (2004)

Schotting, R.J., Moser, H., Hassanizadeh, S.M.: High-concentration-gradient dispersion in porous media: experiments, analysis and approximations. Adv. Water Resour. 22(7), 665-680 (1999)

Scheidegger, A.E.: General theory of dispersion in porous media. J. Geophys. Res. 66, 3273-3278 (1961)

Simmons, C.T.: Variable density groundwater flow: From current challenges to future possibilities. Hydrogeol. J. 13, 116-119 (2005)

Starke, B.: Experimental and numerical investigations of macrodispersion of density-dependent flow and transport in stochastic porous media. PhD- Dissertation (in German), University Kassel, 199 pp (2005)

Starke, B., Koch, M.: Laboratory experiments and Monte Carlo simulations to validate a stochastic theory of density-dependent macrodispersion, CMWRXVI (2006)

Thiele, M.: Gravity affected lateral dispersion and diffusion in a stationary horizontal porous medium shear flow. Transp. Porous Media 26, 553 (1997)

Thiele, M.: Stationary transverse diffusion in a horizontal porous medium boundary-layer flow with concentration-dependent fluid density and viscosity. Transp. Porous Media 36, 341-355 (1999)

Tompson, A.F.B., Gelhar, L.W.: Numerical simulation of solute transport in three-dimensional, randomly heterogeneous porous media. Water Resour. Res. 26(10), 2541-2562 (1990)

Watson, S.J., Barry, D.A., Schotting, R.J., Hassanizadeh, S.M.: On the validity of Darcy's law for stable high-concentration displacements in granular porous media. Transp. Porous Media 47, 149-167 (2002a)

Watson, S.J., Barry, D.A., Schotting, R.J., Hassanizadeh, S.M.: Validation of classical density-dependent solute transport theory for stable, high-concentration gradient brine displacements in coarse and medium sands. Adv. Water Resour. 25, 611-635 (2002b)

Welty, C., Gelhar, L.: Stochastic analysis of the effect of fluid density and viscosity variability on macrodispersion in heterogeneous porous media. Water Resour. Res. 27(8), 2061-2075 (1991)

Welty, C., Kane, III., A.C., Kaufman, L.J.: Stochastic analysis of transverse dispersion in density-coupled transport in aquifers. Water Resour. Res. 39(6), 1150 (2003)

Yates, S.R.: An analytical solution for one-dimensional transport in heterogeneous porous media. Water Resour. Res. 26(10), 2331-2338 (1990) 